\title{
Feasibility study of a high voltage In-Service- Tests on the superconducting magnet system of Wendelstein 7-X
}

\author{
F. Füllenbach, Th. Rummel, Th. Mönnich
}

\begin{abstract}
During the energizing and de-energizing process of the Wendelstein 7-X magnet system, the forces induced in the superconducting coils could lead to stresses in the insulation of the coils, the interconnecting bus system, the current leads and the mechanical support structure. Such stress could cause a ground fault that would not be detected during normal operation. In normal condition the voltage across a coil group is halved by a middle point grounding system. If a ground fault is present during a fast discharge, the coil group could be stressed with the full voltage of up to $4 \mathrm{kV}$ which could endanger the high voltage integrity of the whole superconducting magnet system.

To prevent such undesirable occasions, the insulation of the superconducting magnet system shall be monitored by a high voltage In-Service-Test. That means that a test voltage shall be applied during the operation of the magnets. Therefore, it is planned to temporarily lift the middle point of the grounding system by a high voltage source of $2,5 \mathrm{kV}$ and to measure the leak current. To evaluate the feasibility of such a system, a simulation model of one coil group has been created in Simplorer ${ }^{\odot}$. Considering detailed parameters of the coil group, the model shall support the evaluation of how the high voltage influences the superconducting magnet system and shall determine the leak currents with and without certain damages in the insulation.

The paper describes the Simplorer ${ }^{\odot}$ model with its different simulation scenarios and summarizes the results and findings..
\end{abstract}

Index Terms- Wendelstein 7-X, fusion reactors, superconducting coils, high voltage test

\section{INTRODUCTION}

$\mathrm{T}$ HE main magnetic field of the W7-X will be created by 50 nonplanar and 20 planar superconducting coils. There are seven coil types with 10 coils of each type. One power supply feeds one coil group of ten coils. The superconducting magnets are built upon an epoxy resin impregnated winding pack that is covered by a conductive coating. The conductive coating is in one point electrically connected to the grounded coil casing in which the winding pack is embedded and therefore gives a predefined resistive connection to ground.

During normal operation even significant damage to the coil insulation will not lead to any faulty behavior of the coil as the leak current will flow through the conductive coating and/or the coil casing before reaching ground potential. This path will

Manuscript received 9 September 2011.

F. Füllenbach, Th. Rummel, Th. Mönnich are with the Max-Planck-Institut für Plasmaphysik, Euratom Association, Teilinstitut Greifswald, Wendelsteinstr. 1, D-17489 Greifswald, Germany (corresponding author phone: $+493834 \quad 88$ 2776; fax: $+49 \quad 3834 \quad 88$ 2709; e-mail: frank.fuellenbach@ipp.mpg.de). be of a much higher resistance than the superconducting coil group. Therefore, the ground fault could easily stay unnoticed until a high voltage (HV) e.g. during a fast discharge is applied to the coils. The coil group is fed by one power supply and it is connected to ground via middle point connection between the feeding bus bars. A ground fault due to a damaged insulation can lead to a much higher (in the worst case twice). These insulations have not been tested with such an increased HV. A high voltage test system called the "InServe-Test" (IS-Test), that can operate while the magnets are energized, is supposed to create large enough leak currents to identify such ground faults and trigger safety measures before the coil system gets endangered. A simulation model of the coil group with all relevant connections to ground including a power supply shall be generated to test the behavior of the coil group during the IS-Test. A voltage level of $2.5 \mathrm{kV}$ has been chosen according to the expected maximum voltage during a fast discharge. For applying the voltage two different technologies have been considered:

- Adding a DC HV source that can be switched into to the ground path. DC voltage is easy to handle and will not create any undesired capacity or inductive effects during the flat top. On the downside it will be necessary to open the ground path to add the HV source into the ground path. This will leave the system disconnected to ground during the switching sequence

- Using an AC HV source that induces an AC voltage over inductive coupling into the ground path. The AC source can be added to the ground path without opening the circuit while the coils are operated. Nevertheless, the AC voltage will create capacitive currents in the insulation of the coils. Decreasing the frequency could be a way to minimize the problem, but this will also lower the inductive coupling of the HV source to the ground path.

\section{The Simulation Model}

The two main components of the simulation model will be the coil group and the power supply. The HV source will be included in the power supply model. The high current transmission lines will be included as well as the connections to ground via the water cooling of the transmission line. 


\section{A. The Power Supply}

Each of the seven power supplies is a twelve pulsing thyristor rectifier based upon four M3 groups in parallel. The output values are $\pm 30 \mathrm{Vdc}$ and $20 \mathrm{kA}$ [1]. Since the function of the rectifier is not supposed to be relevant for the simulation (there is no feedback to the power supply), it will be modeled by a simple current source (PSM). The DC output of the rectifier is grounded symmetrically over two resistors with 50 $\mathrm{k} \Omega$ each (Rgrd1 and Rgrd2). The connection of the middle point of the two resistors to the ground (Rgrd3) will be the path in which the high voltage source is going to be included. Figure 1 shows the simulation model for the power supply and the transmission line up to the feedthroughs of the coil group. In this particular figure the high voltage source is a DC source (V1) included in the grounding path without an additional switch gear. Resistors are added for the connection to the ground via the cooling water $\left(\mathrm{Rw}_{\mathrm{n}}\right)$. Values of the resistors are derived from the cooling channel length and the conductivity of the cooling water which can vary between $1 \mu \mathrm{S} / \mathrm{cm}$ to 10 $\mu \mathrm{S} / \mathrm{cm}$. For the simulations the values of $1 \mu \mathrm{S} / \mathrm{cm}, 5 \mu \mathrm{S} / \mathrm{cm}$ and $10 \mu \mathrm{S} / \mathrm{cm}$ have been chosen. For completion the resistances representing the current bus bars $\left(\mathrm{Rss}_{\mathrm{n}}\right)$, the feed through to the cryostat $\left(\mathrm{Rsz}_{\mathrm{n}}\right)$ and the connections between the grounding resistors and the bus bars $\left(\operatorname{Re}_{\mathrm{n}}\right)$ have been added to the model as well. They will play a minor role as the grounding resistances $\left(\operatorname{Rgrd}_{n}\right)$ will dominate the ohmic behavior of the circuit.

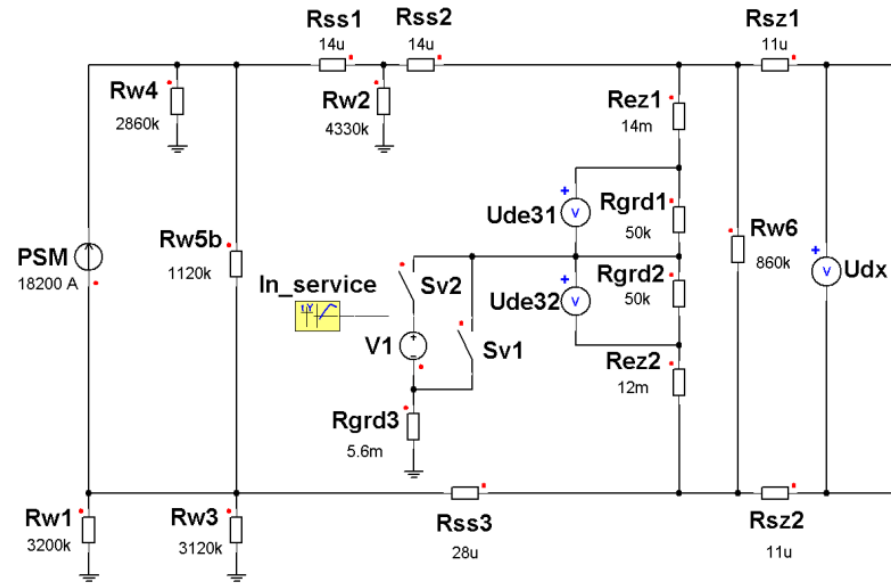

Fig. 1. Model of the power supply circuit with grounding resistances $\operatorname{Rgrd}_{n}$ and interconnections $\mathrm{Re}_{\mathrm{n}}$, cooling water $\mathrm{Rw}_{\mathrm{n}}$, current bus bars $\mathrm{Rss}_{\mathrm{n}}$. Numbers beside give the values in ohm. Furthermore the power supply module PSM as a current source and the DC HV source V1 with the corresponding switch gear Sv1 and Sv2 and the ramp up function In_service.

\section{B. The coil group}

The coil group is comprised of ten individual coils with similar parameters. Fig 2 show three of three coils in series connected. Each coil is divided into two inductances $L_{n}$ representing half of the coil with $50.3 \mathrm{mH}$. The four resistors $\mathrm{R}_{\mathrm{n}}$ for each coil represent the input and output connections with $2 \mathrm{n} \Omega$ and the interlayer connection for each half of the coil with $2.5 \mathrm{n} \Omega$. The insulation to the coil casing is simulated by two RC combinations with $\mathrm{Ri}_{\mathrm{n}}$ and $\mathrm{C}_{\mathrm{n}}$ each forming half of the coil insulation with a cumulated resistance of $1 \mathrm{G} \Omega$ and a capacitance of $90 \mathrm{nF}$. Another resistor $\mathrm{RLL}_{\mathrm{n}}$ of $100 \mathrm{k} \Omega$ simulates the conductive coating of the winding pack which is then connected to coils' casing. The interconnection bus bar system is also displayed with its insulation resistance Rbiso $_{n}$ of $1 \mathrm{G} \Omega$ and its conductive coating $\mathrm{RBLL}_{\mathrm{n}}$ of $200 \mathrm{k} \Omega$. Although the capacitance of the bus bar insulation is unknown, it is supposed to be negligible with respect to the capacitance of the coil insulation and therefore not included.

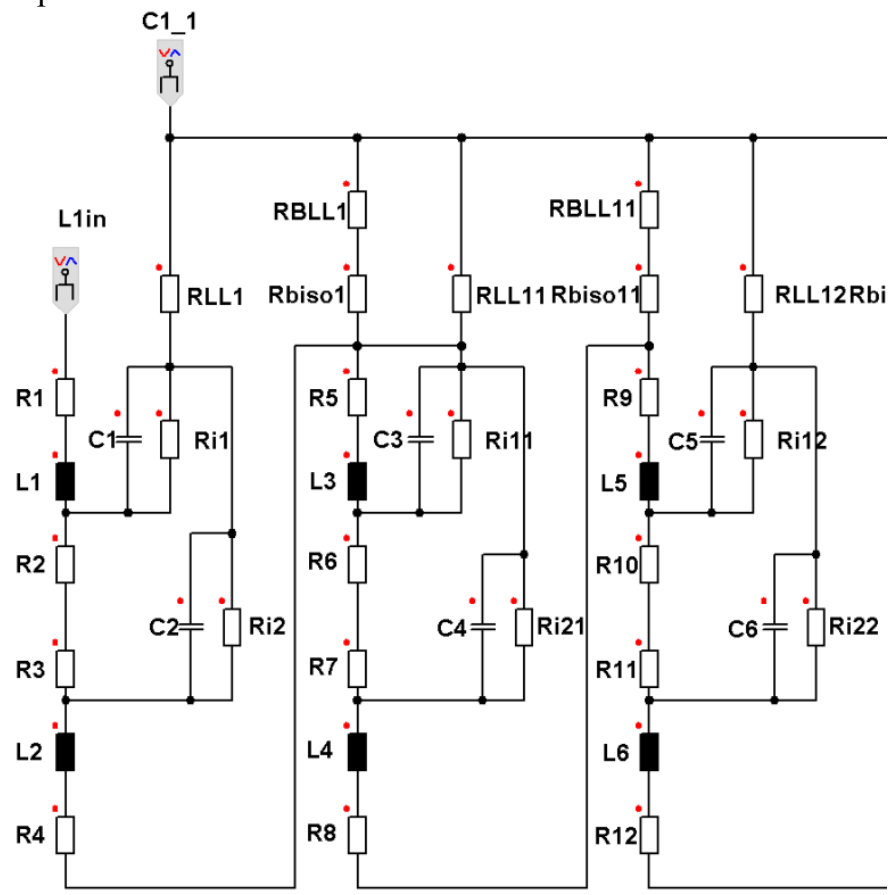

Fig. 2. Model of coil group shows three coils in series with the interconnecting bus. $L_{n}$ is of half the coil inductance, $R_{n}$ represents the interconnections, $\mathrm{Rll}_{\mathrm{n}}$ and $\mathrm{RBLL}_{\mathrm{n}}$ the conductive coating of the coil and the bus system, $\mathrm{C}_{\mathrm{n}}$ and $\mathrm{Ri}_{\mathrm{n}}$ the coil insulation and $\mathrm{RBiso}_{\mathrm{n}}$ the bus insulation

The electrical parameters for the coil insulation have been taken from the results of the coil high voltage tests during their fabrication. While the resistance is an easy value to obtain, the capacitance had to be calculated from the measured leak current while ramping up the test voltage. In order to validate the single coil model, the HV test has been performed on the coil model with regards to the measured leak currents. Table 1 shows that the measured values do fit very well with values obtained from the simulation.

TABLE 1 MARGIN REQUIREMENTS

\begin{tabular}{ccc}
\hline $\begin{array}{c}\text { Test voltage } \\
{[\mathbf{k V}]}\end{array}$ & $\begin{array}{c}\text { Leak current simulated } \\
{[\boldsymbol{\mu A} \mathbf{A}]}\end{array}$ & $\begin{array}{c}\text { Leak current measured } \\
{[\boldsymbol{\mu A} \mathbf{A}]}\end{array}$ \\
\hline 6 & 9,6 & 8 \\
9,1 & 14,5 & 13,8 \\
\hline \hline
\end{tabular}

The values for the conductive coating are based upon the maximum values which have been specified to the supplier. Tests have shown that these values can be exceeded locally but the integral values are supposed to be lower which will aid the detection of leak currents.

\section{SimUlations}

The simulations have been done in three major steps. In a first step, the influence of the IS-Test on a normal operating magnet system has been evaluated. In a second step, different 
ground faults in the coil insulation were introduced under normal operation without any high voltage. Finally, the HV have been added to a normal operating system with different ground faults. The tests were initially done with a DC HV source and then need to be repeated with an AC HV source.

\section{A. Normal operation with high voltage test and without ground fault}

During normal operation the power supply provides a constant current of $18 \mathrm{kA}$ to the coil group. The DC high voltage will then be ramped up within $1 \mathrm{~s}$ or $2 \mathrm{~s}$. It is expected that a very small current will develop due to the capacitance of the coil insulation during ramp up and ramp down of the high voltage. Additionally, a leak current over the cooling water is to be expected depending on the water conductivity. Figure 3 shows the overall leak current to ground with respect to the high voltage. This leak current is the basis for evaluating the results of the test and for the later system to decide whether the insulation is still in order or not.

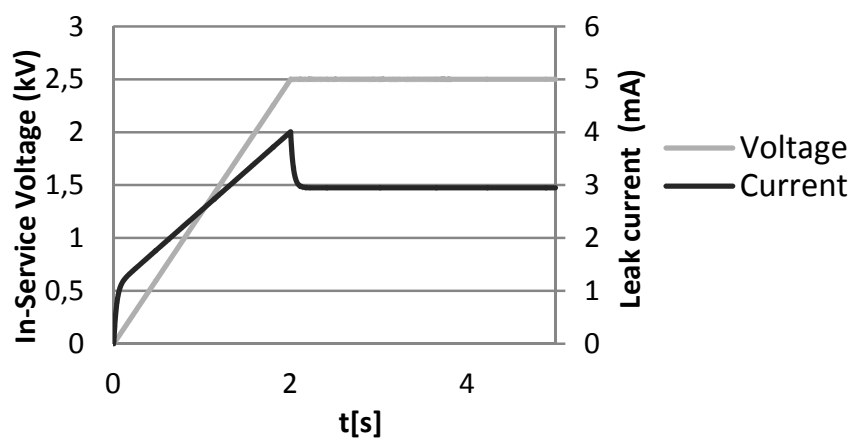

Fig. 3. Leak current during IS-test with an In-Service Voltage of $2.5 \mathrm{kV}$ and a ramp up time of $2 \mathrm{~s}$

To clarify the impact of the In-Service-Test on the operation of the Power supply the following scenarios have been evaluated with and without In-Service-test as well:

- $\quad$ Ramping up the current with max $\frac{d i}{d t}$ using a voltage source as power supply. Voltages and currents over the grounding resistors of the power supply have been observed to assure that there is no influence on the normal operation.

- Fast discharge of the coil group. A switching circuit for the fast discharge on a dump resistor has been added to the model. The currents and voltage over the switches and the resistor have been observed in order to check that there is no influence on the normal operation.

As expected, the In-Service-Test had no influence on the operation of the power supply and the fast discharge circuit

\section{B. Normal operation with ground fault and without IS-Test}

The two resistances $\mathrm{Ri}_{\mathrm{n}}$ (see Figure 2) for the insulation of one coil are decreased to $5 \mathrm{M} \Omega, 1 \mathrm{M} \Omega, 500 \mathrm{k} \Omega$ and $100 \mathrm{k} \Omega$ representing the ground fault. As the coil group is superconducting and resistive connections e.g. in the joints are still in a low $\mathrm{n} \Omega$ range, no measurable leak current should flow over the ground fault. Also adding the ground fault to different coils should not have any influence on the behavior due to the superconductivity of the whole coil group. In figure 4 the currents over the two grounding resistors Rgrd1 and Rgrd 2 are shown. At $t=100 \mathrm{~ms}$ the ground fault occurs and the currents only change in a range of about $150 \mathrm{nA}$. The ground fault detection of the power supply will measure the two voltages over the grounding resistors and compare them. With the given difference current the corresponding voltage is about $7.5 \mathrm{mV}$. This voltage level will stay undetected by the standard ground fault detection system.

\section{Diagrammtitel}

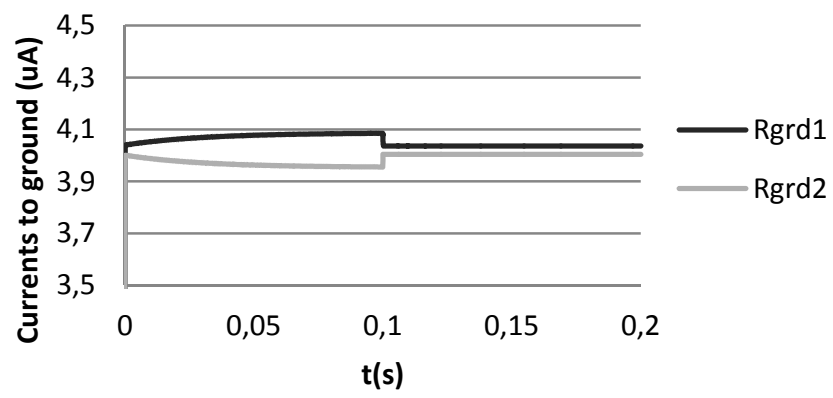

Fig. 4. Currents over grounding resistors when ground fault occurs

\section{Normal operation with ground fault and In-Service-Test}

The tests described under 3.B will be repeated and the IS-Test will be applied to the system. The leak currents that will stay after the flattop of the $\mathrm{HV}$ is reached $\left(\mathrm{I}_{\mathrm{gf}}\right)$, are the decisive values to determine if the system can detect a ground fault. To evaluate the sensitivity of the system these leak currents will be divided by the associated leak current $\left(\mathrm{I}_{\mathrm{g}}\right)$ gained during the test discussed in 3.A. The result of this ratio is called the sensitivity of the detection. Reaching a sensitivity of two is supposed to be necessary to safely detect a ground fault. Figure 5 shows the result for all leak currents of the different ground faults with respect to the different water qualities and the resulting sensitivity factor Igf/Ig. It becomes evident that beside the resistance of the ground fault itself, the water quality has a significant impact on the sensitivity of the ISTest. But in general it can be concluded that the IS-Test will be feasible.

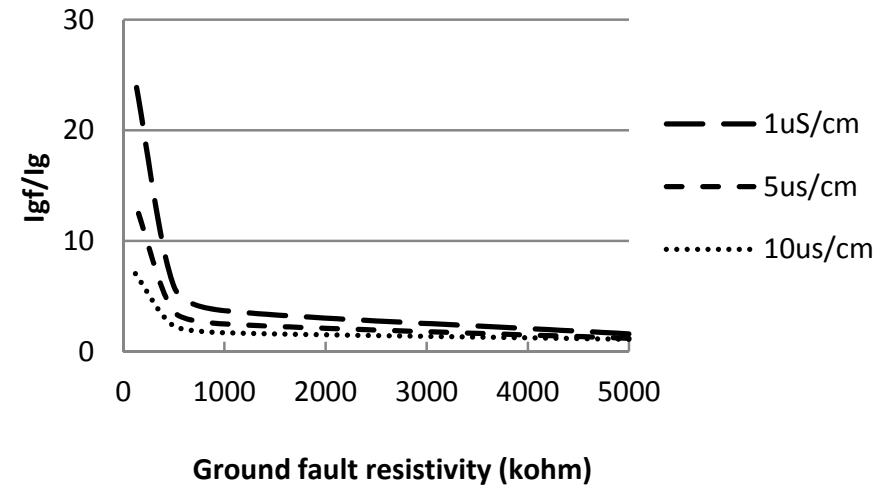

Fig. 5. Sensitivity of the ground fault detection based upon the relation of Igf/Ig 


\section{Simulations with an AC high voltage source}

Figure 6 shows the modified part of the simulation model. A transformer (TWT1) is added to the circuit with the secondary winding connected into the ground path. The transformer is designed to be fed by $230 \mathrm{~V} 50 \mathrm{~Hz}$ (E1) and to deliver a 2.5 $\mathrm{kV}$ peak voltage at the secondary winding. Furthermore, the current source representing the power supply (PSM) needs to be replaced by a voltage source (PSMV) as the current source reacted sensitively to the induced $\mathrm{AC}$ voltage and started oscillating. Repeating the first test as described in 3.B shows the drawback of using the AC HV source: due to the parasitic capacitance of the coil insulation an alternating load current of $70 \mathrm{~mA}$ is generated by the voltage. Now looking at the possible leak currents that can occur due to a ground fault, the circuit can be simplified to the two grounding resistors Rgrd1 and Rgrd2 assuming that the ground fault has not further resistance. Then these two resistors are connected in parallel and the $2.5 \mathrm{kV}$ will feed a $25 \mathrm{k} \Omega$ resistance resulting in a 100 $\mathrm{mA}$ current. This is the theoretical maximum leak current that can occur. Practically, the value will be much lower therefore the relation between the operational leak current and ground fault current is too low to safely detect the ground fault. By lowering the frequency of the In-Service-Test voltage (E1) the leak current will decrease only slightly. At $10 \mathrm{~Hz}$ it will be still $60 \mathrm{~mA}$. Therefore it was decided at this point to drop the solution with an AC HV source.

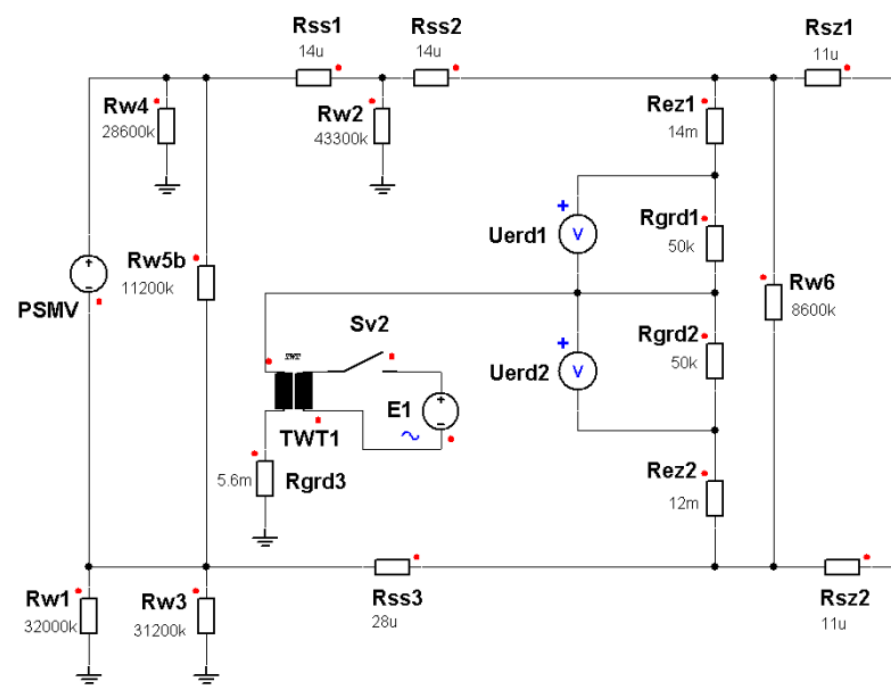

Fig. 6. Model with AC Source with grounding resistances $\operatorname{Rgrd}_{n}$ and interconnections $\mathrm{Re}_{\mathrm{n}}$, cooling water $\mathrm{Rw}_{\mathrm{n}}$, current bus bars $\mathrm{Rss}_{\mathrm{n}}$. Numbers beside give the values in ohm. Furthermore the power supply module PSMV as a voltage source and the AC HV source E1 with the interconnecting transformer TWT1 and the corresponding switchgear Sv2

\section{IMPLICATIONS AND FURTHER PROCEDURE}

To establish an IS-Test system for the superconducting magnet system of W7-X certain boundary conditions have to be considered. The chosen $2.5 \mathrm{kV}$ is a suitable voltage level to detect ground faults and should not be reduced. It has to be assured that the whole power supply system is designed to withstand this voltage against ground. Design documents of the plant have to be checked carefully and additionally tests need be performed over the system. The cooling water quality will differ during operation. Nevertheless, it needs to be checked if the conductivity can be kept at the lower margin with reasonable effort. Furthermore, the actual value needs to be monitored and included in the evaluation of the In-ServiceTest. For safety reason the operation of the In-Service-Test needs to be limited to the flattop phase of the magnet system. As even the simulations do not show any influence on the ramping up and down or the fast discharge of the coils, it is not considered necessary to have the IS-Test available during these phases. An interlock system has to assure that the ISTest is always stopped and brought to a safe condition whenever a ramping of the current or a fast discharge is triggered. Therefore, the IS-Test should be an integrated and subordinated part of the power supply system also with regards to the control system. Keeping this in mind, the further procedure should be to verify the simulation results in a small scale laboratory test with low voltages. A next step could be the verification of the concept with another power supply already existing at the W7-X site. This test should show that a test voltage at a higher level can be performed without disturbing the power supply and that significant leak currents can give a clue about a ground fault with predefined resistivity. Finally, a prototype of the In-Service-Test circuit needs to be developed and tested at the power supply of the superconducting coils itself.

\section{SUMMARY}

In this paper, simulations to verify the feasibility of an InService-Test system for superconducting magnet system of W7-X have been performed. The simulation models were cross checked with measured data gained during tests with coils and the power supply and have been proven to be reasonable. The results clearly show that such a test system is feasible using DC HV. Simulations with AC HV proved that the obtained results will not help to definitely detect a ground fault. Nevertheless, even the DC HV does not allow detecting a slowly degrading insulation or the location of a ground fault. Comparing the results of different operation scenarios, it will be inevitable to monitor the conductivity of the cooling water which is used to cool the current bus bars during operation and to include the values in the evaluation of the In-Service-Test results. The next steps in further verification of the concept and development of a prototype have been scheduled and are well underway.

[1]Th. Rummel, F. Füllenbach and Th. Mönnich, "Power supplies for the Wendelstein 7-X stellarator", Fusion Engineering and Design, 66-68 (2003), pp. $1115-1118$ 